\title{
Garcin Syndrome Due to Meningeal Carcinomatosis from Gastric Cancer
}

\author{
Kei Terasaki, Chiemi Mizuno, Shinsaku Fujiishi, Shohei Amioka, Takahiro Nonaka, \\ Junichiro Itani, Yusuke Takahashi, Takahiro Miura, Tomoko Yasufuku, Hirohisa Oya, \\ Takayuki Katayama, Kotaro Okuda, Junko Matsumoto, Yasuhide Mitsumoto, Naoki Sawai, \\ Masayuki Mizuno, Toshihide Shima and Takeshi Okanoue
}

\begin{abstract}
:
A 58-year-old woman presented to our hospital with complaints of dysphagia. Esophagogastroduodenoscopy showed an esophagogastric junction tumor with multiple duodenal intramural metastases, and computed tomography showed peritoneal metastasis. In the middle of her fourth cycle of chemotherapy, she displayed symptoms of a left-sided multi-cranial nerve palsy. She was diagnosed with Garcin syndrome caused by meningeal carcinomatosis from gastric cancer based on the results of gadolinium-enhanced brain magnetic resonance imaging and cytology of the cerebrospinal fluid. It is important not to overlook meningeal irritation symptoms or paralysis of cranial nerves and to consider the possibility of Garcin syndrome caused by meningeal carcinomatosis.
\end{abstract}

Key words: garcin syndrome, meningeal carcinomatosis, gastric cancer

(Intern Med 60: 855-858, 2021)

(DOI: 10.2169/internalmedicine.5797-20)

\section{Introduction}

Meningeal carcinomatosis from gastric cancer is a relatively rare condition, and there has been only one reported case of Garcin syndrome caused by meningeal carcinomatosis from gastric cancer. Garcin syndrome is characterized by the presence of unilateral palsies owing to multiple-cranial nerve involvement. This syndrome comprises an absence of motor or sensory signs of the limbs and no signs of intracranial hypertension (1).

We herein report a rare case of Garcin syndrome in a patient caused by meningeal carcinomatosis from gastric cancer.

\section{Case Report}

A 58-year-old woman with no medical history had dysphagia for 2 months before being admitted to our hospital. This symptom gradually worsened over time.
Esophagogastroduodenoscopy showed an esophagogastric junction (EGJ) tumor with circumferential stenosis with multiple erosions at the duodenal bulb to the superior duodenal angle (Fig. 1A-C). The histopathological diagnosis from a biopsy of the EGJ tumor was signet ring cell carcinoma (Fig. 2), which was the same as the histopathological diagnosis from a biopsy of the duodenum; hence, she was diagnosed with EGJ cancer with multiple duodenal intramural metastases.

Contrast-enhanced abdominal computed tomography (CT) showed peritoneal metastasis and bilateral hydronephrosis caused by cancer exclusion of the urinary tract. Thus, she was diagnosed with stage IV gastric cancer and began chemotherapy ( $\mathrm{S}-1+$ cisplatin). After the third cycle, chest and abdominal CT and esophagogastroduodenoscopy showed a slight reduction in the cancer volume.

In the middle of the fourth chemotherapy cycle, she started complaining of a headache and nausea. No abnormalities were detected on brain CT the day after her headache and nausea appeared. Therefore, it was thought to be a

Department of Gastroenterology and Hepatology, Saiseikai Suita Hospital, Japan 


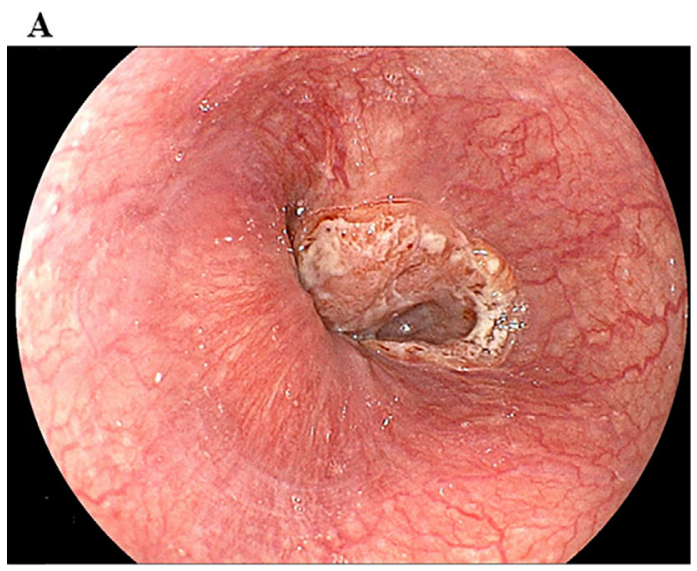

B

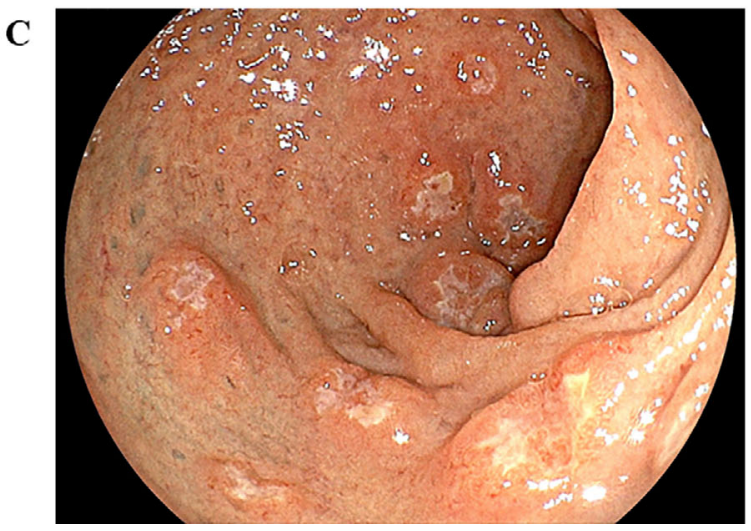

Figure 1. Esophagogastroduodenoscopy findings on admission. Esophagogastroduodenoscopy showing (A, B) an esophagogastric junction (EGJ) tumor with circumferential stenosis of the EGJ, and (C) multiple erosions at the duodenal bulb to the superior duodenal angle.

A

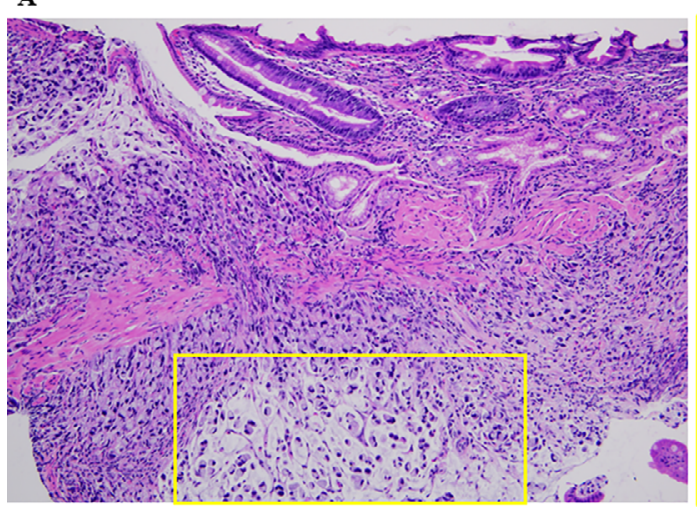

B

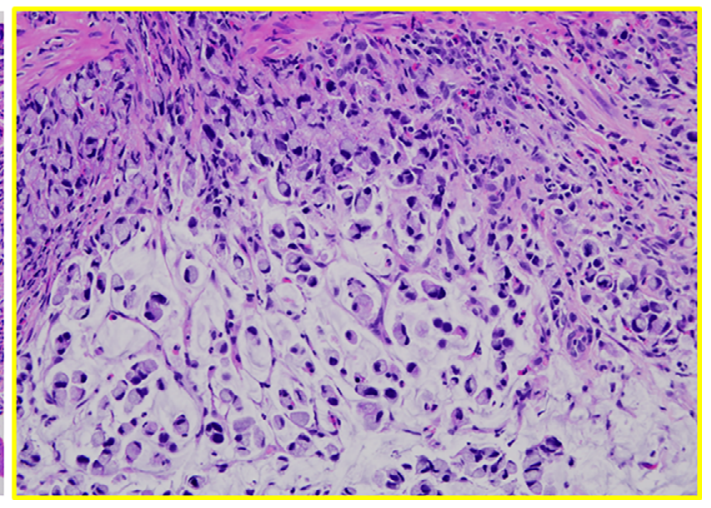

Figure 2. Histopathological findings of a biopsy from the EGJ. Microscopic examinations revealing signet ring cell carcinoma with small collective nests of signet ring cells. (A) Low-power field, and (B) high-power field.

migraine headache, and she was prescribed a nonsteroidal anti-inflammatory drug (NSAID) and a 5-hydroxytryptamine (5-HT) receptor agonist. Her migraine headache temporarily disappeared but relapsed as soon as the medicinal effect expired. Five days later, a further evaluation by gadoliniumenhanced brain magnetic resonance imaging (MRI) showed no abnormality. Laboratory examinations revealed anemia (hemoglobin $8.2 \mathrm{~g} / \mathrm{dL}$ ) and hypoalbuminemia (total protein $5.6 \mathrm{~g} / \mathrm{dL}$, albumin $3.5 \mathrm{~g} / \mathrm{dL}$ ), but there was no indication of an inflammation reaction (C-reactive protein $0.1 \mathrm{mg} / \mathrm{dL}$ ).

Thirty-five days later, she displayed hyperesthesia in the left ophthalmic nerve, left lagophthalmos, and left eyelash sign, and the left corner of her mouth dropped suddenly, but there were no signs of quadriplegia. Gadolinium-enhanced brain MRI and magnetic resonance angiography (MRA) were immediately performed again because cerebral infarction or brain metastasis of gastric cancer was suspected. MRI and MRA showed no evidence of cerebral infarction 


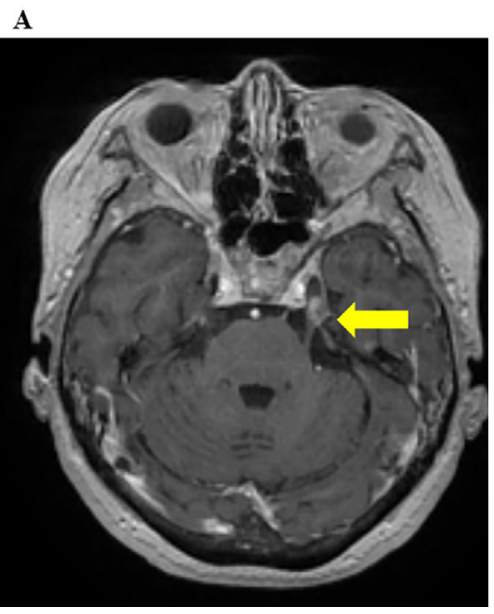

B

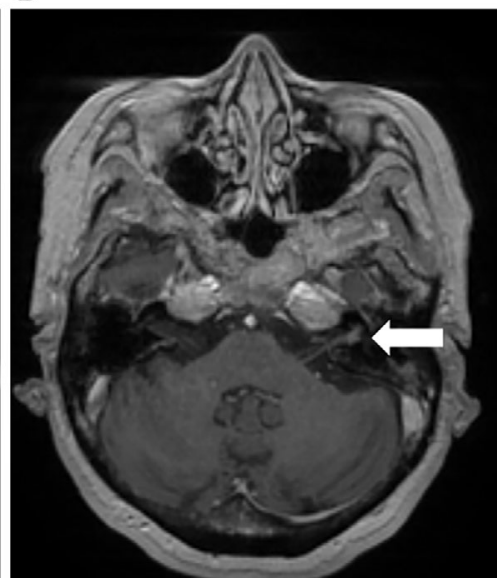

Figure 3. Gadolinium-enhanced brain magnetic resonance imaging findings. Brain enhanced magnetic resonance imaging showing abnormal dense staining in the trigeminal nerve (A: yellow arrow) and facial nerve (B: white arrow) on gadolinium-enhanced T1-weighted imaging.

but did show abnormal dense staining on gadoliniumenhanced T1-weighted imaging along the trigeminal nerve and facial nerve (Fig. 3). Furthermore, this left-sided trigeminal nerve and facial nerve palsy was followed by left eyeball adduction, curtain sign, loss of the gag reflex, articulation disorder, taste disorder, left deviation of the tongue tip, and left hearing disorder that developed progressively over two days. These symptoms were thought to have been caused by left-sided cranial nerve palsy from cranial nerve V, VI, VII, VIII, IX, X, and XII involvement.

For a further examination, cerebrospinal fluid (CSF) puncture was performed, and the CSF examination revealed a normal initial pressure, an increased cell count $(15 / \mu \mathrm{L})$, a high protein level $(57.7 \mathrm{mg} / \mathrm{dL})$, and a normal glucose level $(62 \mathrm{mg} / \mathrm{dL})$. Viable bacteria could not be cultured from the CSF. Cytology of the CSF showed signet ring cells, findings that were the same as those obtained via histology of the EGJ cancer. Forty days later, she was diagnosed with Garcin syndrome due to meningeal carcinomatosis from gastric cancer.

She underwent whole-brain radiation therapy (2.5 Gy/day) at 45 days and received this therapy 4 times. She was scheduled to be transferred to another hospital for intrathecal administration of anticancer drugs for additional therapy, but she died at 49 days.

\section{Discussion}

The most common causes of multiple cranial nerve palsy are Tolosa-Hunt syndrome, sarcoidosis, Guillain-Barré syndrome, hypertrophic pachymeningitis, infections - such as fungal infection or tuberculosis - and malignancies (2). We herein report a rare case of Garcin syndrome presenting with progressive unilateral paralysis of cranial nerves due to meningeal carcinomatosis from gastric cancer. To our knowledge, only one case of Garcin syndrome due to basal skull metastasis of gastric cancer has been previously re- ported by Harada et al. (3). With respect to the pathogenesis of Garcin syndrome, epidural tumors on the skull base are thought to develop unilateral, sequential cranial neuropathies by propagating along the dura rather than within the skull.

CT and MRI, especially gadolinium-enhanced MRI, are useful for the diagnosis. A characteristic feature is the presence of contrast findings along the cerebral surface and gyrus. In this case, initial brain CT and gadoliniumenhanced MRI showed no abnormal findings; however, a second gadolinium-enhanced MRI performed 30 days later revealed meningeal carcinomatosis from gastric cancer. Considering these findings, along with the results of cytology of the CSF and the progressive unilateral paralysis of cranial nerves, she was diagnosed with Garcin syndrome due to meningeal carcinomatosis from gastric cancer.

Meningeal carcinomatosis is the infiltration of metastatic malignant cells into the meninges from a distant and solid tumor (4). A large autopsy study reported that the incidence of meningeal carcinomatosis is approximately 5-8\% in patients with cancer $(4,5)$. The primary lesion is commonly from melanoma (23\%), lung cancer (9-25\%), and breast cancer $(5 \%)(4,6)$. Only an estimated $0.16-0.69 \%$ of patients with gastric cancer develop meningeal carcinomatosis (7-9). The histological types of gastric cancer that develop meningeal carcinomatosis are predominantly poorly differentiated adenocarcinoma and signet ring cell carcinomas.

The strategy for treating Garcin syndrome due to meningeal carcinomatosis in general is whole-brain radiation therapy and the intrathecal administration of anticancer drugs individually or in combination (10-13). Anticancer drugs usually exhibit difficulty penetrating the blood-brain barrier (13); therefore, therapeutic effects can be achieved by intrathecal administration with a far smaller dose of anticancer drugs than systemic chemotherapy.

Regarding anticancer drugs, methotrexate (MTX) in combination with cytarabine (AraC) is preferable to MTX alone 
for intrathecal chemotherapy $(13,14)$; however, because these anticancer drugs may cause leukoencephalopathy, other anticancer drugs, along with varied timings of administration, can be adopted to achieve a better outcome $(13,14)$.

The median survival of patients with meningeal carcinomatosis is reported to be only six weeks without any treatment, or two to three months with systemic therapy, such as radiation therapy or intrathecal chemotherapy $(4,8,15)$. Very few patients with gastric cancer develop Garcin syndrome due to meningeal carcinomatosis, making this syndrome difficult to diagnose. In this case, several days and tests were required to diagnose her series of symptoms as Garcin syndrome due to meningeal carcinomatosis. It was hypothesized that the headache and nausea occurred due to meningeal carcinomatosis from gastric cancer, and subsequently Garcin syndrome had developed, followed by paralysis of the cranial nerves.

Although whole-brain radiation treatment was started immediately after the diagnosis, she ultimately died four days later. If she had been diagnosed earlier, she may have survived a little longer.

The histological types of gastric cancer that develop meningeal carcinomatosis are predominantly poorly differentiated adenocarcinoma and signet ring cell carcinomas, and the dominant metastatic form is disseminated metastases of peritoneal seeding or lymphatic metastasis. Therefore, for patients with these characteristics of gastric cancer, when persistent headache or cranial neuropathies appear, it is important to consider the possibility of meningeal carcinomatosis and Garcin syndrome from gastric cancer and to consider performing CSF puncture for further examination even if CT and MRI show no abnormalities initially.

To improve the prognosis of this disease even slightly, it is important not to overlook meningeal irritation symptoms or paralysis of cranial nerves and to explore the diagnosis of Garcin syndrome due to meningeal carcinomatosis. This may lead to an earlier diagnosis and initiation of treatment, thereby slightly extending the survival period.

The authors state that they have no Conflict of Interest (COI).

\section{Acknowledgement}

We thank all the members of the Department of Gastroenterology and Hepatology, Saiseikai Suita Hospital for helping us to perform this study.

\section{References}

1. Fujii M, Kiura K, Takigawa N, et al. Presentation of Garcin syndrome due to lung cancer. J Thoracic Oncol 2: 877-878, 2007.

2. Kawahara Y, Deguchi K, Sato K, et al. Aggressive Garcin's syndrome by acquired cystic disease of kidney-related renal cell carcinoma in a long-term hemodialytic patient. J Neurol Sci 355: 216218, 2015.

3. Harada S, Toya S, Iisaka Y, Ohtani M, Nakamura Y. Basal skull metastasis of stomach cancer presenting with Garcin's syndrome. No Shinkei Geka 15: 765-769, 1987 (in Japanese, Abstract in English).

4. Grossman SA, Krabak MJ. Leptomeningeal carcinomatosis. Cancer Treat Rev 25: 103-119, 1999.

5. Kimura A, Takahashi Y, Mizutari K, Tsujimoto H, Nakanishi K, Shiotani A. A case of gastric meningeal carcinomatosis involving bilateral hearing loss: the difference between clinical images and autopsy findings. J Int Adv Otol 15: 333-336, 2019.

6. Pavlidis N. The diagnostic and therapeutic management of leptomeningeal carcinomatosis. Ann Oncol 15: iv285-iv291, 2004.

7. Kim M. Intracranial involvement by metastatic advanced gastric carcinoma. J Neuro-Oncol 43: 59-62, 1999.

8. Wasserstrom WR, Glass JP, Posner JB. Diagnosis and treatment of leptomeningeal metastases from solid tumors: experience with 90 patients. Cancer 49: 759-772, 1982.

9. Jiao XD, Ding C, Zang YS, et al. Rapid symptomatic relief of HER2-positive gastric cancer leptomeningeal carcinomatosis with lapatinib, trastuzumab and capecitabine: a case report. BMC Cancer 18: 206, 2018.

10. Bruno MK, Raizer J. Leptomeningeal metastases from solid tumors (meningeal carcinomatosis). Cancer Treat Res 125: 31-52, 2005.

11. Chowdhary S, Chamberlain M. Leptomeningeal metastases: current concepts and management guidelines. J Natl Compr Netw 3: 693-703, 2005.

12. Aiello-Laws L, Rutledge DN. Management of adult patients receiving intraventricular chemotherapy for the treatment of leptomeningeal metastasis. Clin J Oncol Nurs 12: 429-435, 2008.

13. Yamasaki T, Fukui H, Sei H, et al. Efficacy of intrathecal MTX/ Ara-C combined with systemic chemotherapy in a gastric cancer patient with meningeal carcinomatosis. Intern Med (Tokyo, Japan) 55: 609-611, 2016.

14. Kim DY, Lee KW, Yun T, et al. Comparison of intrathecal chemotherapy for leptomeningeal carcinomatosis of a solid tumor: methotrexate alone versus methotrexate in combination with cytosine arabinoside and hydrocortisone. Japanese J Clinical Oncol 33: 608-612, 2003

15. Nakashima K, Demura Y, Oi M, et al. Whole-brain radiation and pembrolizumab treatment for a non-small-cell lung cancer patient with meningeal carcinomatosis lacking driver oncogenes led to a long-term survival: a case report. Intern Med (Tokyo, Japan) 59: 1433-1435, 2020.

The Internal Medicine is an Open Access journal distributed under the Creative Commons Attribution-NonCommercial-NoDerivatives 4.0 International License. To view the details of this license, please visit (https://creativecommons.org/licenses/ by-nc-nd/4.0/).

(C) 2021 The Japanese Society of Internal Medicine Intern Med 60: 855-858, 2021 